\title{
AOIR
}

Selected Papers of \#AoIR2020:

The 21 st Annual Conference of the

Association of Internet Researchers

Virtual Event / 27-31 October 2020

\section{DISCONNECTION: DESIGNS AND DESIRES}

\author{
Tero Karppi \\ University of Toronto \\ Aleena Chia \\ Simon Fraser University \\ Airi Lampinen \\ Stockholm University \\ Zeena Feldman \\ King's College London \\ Michael Dieter \\ University of Warwick \\ Pedro Ferreira \\ IT-University of Copenhagen \\ Alex Beattie
Victoria University of Wellington

\section{Introductory Statement}

"We can disconnect, risk less prosperity and hope jobs that are lost come back. Or we can connect more, try to do more great things, try to work on even greater prosperity, and then work to aggressively share that prosperity with everyone," Mark Zuckerberg the Facebook CEO reportedly stated in a keynote speech at the APEC, in 2016 after the election of Donald Trump as the President of the USA (AFP 2016). Zuckerberg's statement suggests that our prosperity, conditions that make a good life, are now contingent on social media connectivity. An opposite view to Zuckerberg's statement comes from the so-called "techlash;" a criticism by journalists, users, and politicians who are asking social media platforms to account for turning users into addicts, destroying democracy, and killing privacy. In these views, the designs of social media do not bring

Suggested Citation (APA): Karppi, T., Chia, A., Lampinen, A., Feldman, Z., Dieter, M., Ferreira, P, \& Beatie, A. (2020, October 28-31). Disconnection: Designs and Desires. Panel presented at AolR 2020: The 21th Annual Conference of the Association of Internet Researchers. Virtual Event: AolR. Retrieved from http://spir.aoir.org. 
a better world for everyone but quite on the contrary, they erode the foundations of our civil society by making us vulnerable to the dissemination of fake news, spreading of political propaganda and manipulation of users through psychological modeling.

The conceptual notion of disconnection is becoming central to how we negotiate the boundaries and conditions of our online existence (Light 2014; Syvertsen 2017; Karppi 2018; Brenner 2019). Instead of simply seeing connection and disconnection as binaries that negate each other, the papers of our panel argue for a more nuanced understanding of how disconnection can be a force that produces new forms of connectivity and vice versa (See Light \& Cassidy 2014). Positioning disconnection as the absolute outside of connectivity would only render it incomprehensible, and that would only serve the purposes of tech companies who want to make the option disappear. Thus, by focusing on the designs and desires of disconnection we aim not to ask the essentialist question of what disconnection is, but to find answers to minor questions such as what process have led to the desires to disconnect; how does something disconnect; when does it disconnect; what does it disconnect; and whose disconnection it is?

The papers of this panel focus on the desires to choose to disconnect and the designs that can either satisfy or suppress those desires. Zeena Feldman examines how people talk about logging on and logging off by analyzing a dataset of interviews and surveys from 477 participants in 35 countries. In this study, many participants framed their networked practices as existing beyond choice. One of the implications of the study is that digital participation has become structurally necessary for users and society, calling into question the conventional frame of user agency. In other words, social media platforms have become utilities in users' daily lives and the more integrated they become with users' everyday, the more important the choice to control what happens online becomes. Tero Karppi's paper picks up this angle and focuses on the discourses of Facebook's new off-Facebook Activity Tool. By using the vocabulary of behavioral economics, the paper argues that while Facebook's builds tools for the users to take control of their privacy and allows them to disconnect their data flows between the platform and the advertisers, the company also nudges the users not to make that choice by setting the defaults for sharing, appealing on users' desires for personalized content, and eventually claiming that if targeted marketing is prevented the web will no longer be free. The third paper looks at how being physically away from the technologically-infused world makes users choose to reconfigure their engagements with technology. By analyzing over 30 interviews, Airi Lampinen and Pedro Ferreira point out that being away directs the attention to what is meaningful and purposeful in connections and these perspectives can be helpful for technology design. Michael Dieter traces the spread of card deck methodologies from Scandinavian participatory design to their prominence in contemporary behavioural and user experience design practice. This paper uses interface criticism to reflect on how card decks in design are used to drive commercial patterns of user engagement, conversion and capture and asks what kind of alternative ensembles and disconnective paths from the current configurations of platform capitalism might emerge by redirecting these formats. Lastly, 
Alex Beattie and Aleena Chia discuss the platformization of disconnection by analyzing complementarities between The Light Phone, a sleek mobile device with minimal "smart" functions and Google's wellbeing experiments, a branded collection of digital and analog applications that playfully frame disconnection as dilettantism. Drawing from developer interviews and promotional materials, this paper proposes that by playing both sides of the techlash, tech companies use the institutionalization of ethics to reinvent the new spirit of capitalism.

\section{References}

AFP, "Facebook's Zuckerberg Urges Post-Trump World Not To 'Disconnect'," The Times of Israel, November 21, 2016, https://www.timesofisrael.com/facebookszuckerberg-urges-post-trump-world-not-todisconnect/.

Brennen B. 2019. Opting Out of Digital Media. Routledge.

Hesselberth P., 2018. "Discourses on Disconnectivity and the Right to Disconnect," New Media \& Society 20(5): 1994-2010.

Karppi T.. 2018. Disconnect: Facebook's Affective Bonds. Minneapolis: University of Minnesota Press.

Light B.. 2014. Disconnecting with Social Networking Sites. Palgrave Macmillan UK.

Light B. and Cassidy E. 2014. 'Strategies for the Suspension and Prevention of Connection: Rendering Disconnection as Socioeconomic Lubricant with Facebook': New Media \& Society 16 (7): 1169-84. https://doi.org/10.1177/1461444814544002.

Syvertsen T. 2017. Media Resistance - Protest, Dislike, Abstention. Palgrave Macmillan. 


\title{
AOIR
}

Selected Papers of \#AoIR2020:

The 21 st Annual Conference of the

Association of Internet Researchers

Virtual Event / 27-31 October 2020

\section{ON QUITTING SOCIAL MEDIA: AGENCY IS A RED HERRING}

\author{
Zeena Feldman \\ King's College London
}

In the UK, we check our smartphones, on average, every 12 minutes (Ofcom 2018: 59). That's 90 screen sessions a day, assuming we sleep 8 hours a night. In the UK, more than three-quarters of the population has at least one social media profile and Facebook alone reaches $90 \%$ of us (ibid., 73). Such pervasive connectivity has its critics. Among them, Sherry Turkle warns that 'we often find ourselves bored because we have become accustomed to a constant feed of connection, information, and entertainment' (Turkle 2015: 4). But beyond condemnation, how can we make sense of the mediated, always-on lives many of us lead? And how can we understand practices of digital disconnection therein?

Today's digital platforms and the habits they enable are riddled with affective contradiction. While social networking sites and smartphone apps may offer convenience and conviviality, they can also act as gateways for anxiety, competitiveness and unwanted obligation. This paper seeks to theorise and empirically unpack the paradoxes and ambivalences around digital participation in order to challenge the normative role of 'choice' which animates many discussions about social media (dis)connection. Tech consumers and analysts routinely claim that if users are dissatisfied with a particular digital service, they can simply stop using it. In other words, the solution is to go cold turkey and 'just say no'. But in this paper, I argue that analysing user experiences, practices and motivations through the lens of individual agency misses the point precisely because that approach profoundly misunderstands how choice operates in contemporary digital culture. The fact that platform monopolies structure today's communications landscape - consider, for instance, that Facebook is the world's most populated country (Clement 2020) and that Facebook owns both Instagram and Whatsapp (Shead 2019) - makes clear that opting out is not a realistic option for many.

This paper's analysis of disconnection practices and its critique of user agency are grounded in the Quitting Social Media project and its dataset of survey results from 477 participants in 35 countries, 155 'audience diaries' from a participatory art installation and 12 in-depth interviews. Quitting Social Media examined the discursive landscape of 
digital connectivity and disconnection. It explored how people talked about the relationship between logging on and logging off. What were the ways users negotiated tensions between the pressures and pleasures of participation? How did they make sense of their continued social media use despite their frequently articulated desires and occasional attempts - to disconnect? And why was disconnection so affectively fraught for participants when, functionally, it seemed like such an easy thing to do? Here, the red herring of agency rears its head.

Indeed, many participants framed their networked practices as existing beyond choice. This was in part because they regarded digital participation as structurally necessary for themselves, their social lives and their economic livelihoods. For many, digital connection was seen as essential because 'every waking moment has become the time in which we make our living, and when we submit even our leisure for numerical evaluation via likes on Facebook and Instagram [...] time becomes an economic resource that we can no longer justify spending on "nothing"' (Odell 2019: 15). At the same time, participants also understood social media beyond the simplistic hyperbole of headlines. Rather than straightforward celebration or condemnation, users narrated their social media entanglements with nuance, habitually addressing the complexities and paradoxes of (dis)connection. It emerged that, for them, disconnecting from a service or a device was not so much a rupture in connectivity as it was a means of enabling future participation. Usage stoppages were thus regarded as practices consistent with digital culture and as deeply embedded therein. Among the data points that most clearly captured this pertain to how participants managed social media apps on their smartphones: $93 \%$ of survey respondents had installed a social media app on their phone, and $85 \%$ later deleting one of these apps. Yet nearly $60 \%$ of respondents eventually re-installed a deleted social app.

The paper concludes by mapping these findings onto the economist Albert Hirschman's (1970) seminal framework for understanding organisational failure and success. In Exit, Voice, and Loyalty, Hirschman finds that consumers respond in one of two ways to perceived deteriorations in a service's quality. They might exercise 'voice' and try to rehabilitate the service by taking communicative action - for example, via petitions, referenda and public consultations. Or, if they have the means to do so, they might 'exit' the failing service and move onto an alternative. Hirschman concluded that 'the greater the availability of exit, the less likely voice will be used'. And this principle was articulated by many of my participants, who said people were free to leave if they weren't happy with a particular platform. For them, individual action - i.e. exit - seemed the only option; no one talked about working together to make systemic change. Yet my participants also acknowledged the impossibility of complete withdrawal from digital connectivity. As such, I explore how Hirschman's strategies of 'voice' and 'exit' can be rehabilitated in the context of beyond choice epistemology, where it is increasingly seen as both necessary and impossible to disconnect. 


\section{References}

Clement, J. 2020. 'Number of monthly active Facebook users worldwide as of 4th quarter 2019'. Statistica. Available at https://www.statista.com/statistics/264810/number-of-monthly-active-facebookusersworldwide.

Hirschman, A. O. 1970. Exit, Voice, and Loyalty: Responses to Decline in Firms, Organizations, and States. Cambridge: Harvard University Press.

Odell, J. 2019. How to Do Nothing: Resisting the Attention Economy. Brooklyn and London: Melville House

Ofcom. 2018. Communications Market Report 2018. Available at https://www.ofcom.org.uk/research-and-data/multi-sector-research/cmr/cmr-2018.

Shead, S. 2019. 'Facebook owns the four most downloaded apps of the decade'. BBC News. Available at https://www.bbc.co.uk/news/technology-50838013.

Turkle, S. 2015. Reclaiming Conversation: The Power of Talk in a Digital Age. New York: Penguin Press. 


\title{
AOR
}

Selected Papers of \#AoIR2020: The 21 st Annual Conference of the Association of Internet Researchers Virtual Event / 27-31 October 2020

\section{OFF-FACEBOOK ACTIVITY}

\author{
Tero Karppi \\ University of Toronto
}

"The future is private," claimed the message on the screen behind Mark Zuckerberg at Facebook's F8 developer conference in 2019. "Privacy", a notion important to users but absent from Facebook's official vocabulary (Vaidhyanathan 2018: 72-73) had suddenly become the slogan of the company's future plans. This increased awareness of privacy was likely a response to the 2018 case of Cambridge Analytica revelations and the 2016 psychographic profiling and targeting of voters based on Facebook data, which gave rise to campaigns such as \#DeleteFacebook demanding that users disconnect their Facebook profiles. On August 20, 2019, the company gave its users the first example of what the more private future could look like in the form of the Off-Facebook Activity tool. The Off-Facebook Activity tool lets the user see a summary of the apps and websites that by default are allowed to send Facebook information about the user's activity. If the user so chooses, they can clear out this information from their accounts. The choice to block the data flows from particular advertisers or businesses does not stop online advertising but the company says it directly impacts the content the user sees: "You'll still see the same number of ads, but the ads you see may be less personalized to you" (Facebook A).

In this paper, which is based on a longer chapter in the forthcoming Undoing Networks book, I will draw on the work of Pepita Hesselberth (2018), who sees disconnection as a process that does not negate connectivity but gives rise to an outside which is immanently present. I argue that the constitution of this outside and its politicization is at the heart of the Off-Facebook Activity tool. By utilizing a framework which mixes approaches from nudge theory and software studies, I will do a close reading on the promotional materials of the Off-Facebook Activity tool; I will map how the tool technically draws borders between the Facebook platform, its users, and advertisers by disconnecting links between them. I also analyze how the discourses that surround the tool nudge the user towards particular forms of exteriority and exclusion. In other words, when the Off-Facebook Activity tool defines Facebook's outside it does it in practice as a function of technology, but also in the surrounding discourses. The limits of the outside are technical, social, and psychological at the same time. 
"The best person to be in control of data is you," the Off-Facebook Activity tool press release declares (Facebook b). The discourses of the Off-Facebook Activity tool nudge the user to evaluate their choice to disconnect through gains and losses encouraging them to keep on using the system rather than blocking it or giving up entirely. Individual control that takes the form of choice highlighted by the promotional materials has a long history in the development of privacy tools. The choice is also a central notion in Richard Thaler and Cass Sunstein's (2008) nudge theory which comes from the field of behavioral economics. The authors argue that human behavior cannot be controlled by relying on rationality in decision-making situations, for example, through providing as much information as possible. Instead, people should be nudged toward certain behaviors by modifying the environment in which choices take place. Nudges ever so slightly modify the environment in order to steer individuals' decisions. Important to the effectiveness of nudge theory then is that individuals stay within the system or environment where they can be influenced: the outside becomes a non-choice.

In my analysis, I focus on four instances where instead of privacy, the nudges of the OffFacebook Activity tool are designed to steer the individuals to choose to stay connected not only with the Facebook platform but also with its practices of targeted and personalized advertising. The first nudge is the choice of default settings: as information scholars among others have demonstrated users tend not to change their defaults (Shah \& Sandvig 2008). The second nudge aims to define the Off-Facebook is a space constituted by third parties such as advertisers. Research however indicates that these third parties are not external to the platform but elementarily integrated with it by Application Programming Interfaces and Software Development Kits; third parties are what financially sustains the operation of the platform (Helmond et al. 2019). Rather than constituting an actual outside the Off-Facebook space camouflages the importance of user data for the company's business by naming the advertisers as outsiders. The third and the fourth nudge are connected to the second one; the promotional materials make a strong argument that if the user does not allow targeted marketing, Facebook and the entire Web will soon no longer be free. By defaulting users, the promotional discourses imply that the user is in debt for the platform and personal data is the repayment. Thus, the third nudge declares that the best way to repay is to not adjust offFacebook activity settings. The fourth nudge exploits users' tendencies for loss aversion a belief that "losses are weighted substantially more than objectively commensurate gains" (Kahneman et al. 1990). In other words, the choice to disconnect and use the offFacebook Activity tool is not selected until it becomes significantly more valuable than connectivity. By placing disconnection and the use of the tool as a risk to the entire free web the outside of connectivity vanishes as an impossibility.

\section{References}

Facebook A. "How Do I Disconnect My Off-Facebook Activity?", Facebook Help Center, accessed January 29, 2020, https://www.facebook.com/help/287199741901674?helpref=faq_content. 
Facebook B. "The Best Person To Be in Control of Data Is You," Facebook, accessed January 28, 2020, https://www.facebook.com/off-facebook-activity.

Helmond A, Nieborg D.B., and van der Vlist F.N. 2019. "Facebook's Evolution: Development of a Platform-as-Infrastructure," Internet Histories 3(2): 123-46.

Hesselberth P. 2018. "Discourses on Disconnectivity and the Right to Disconnect," New Media \& Society 20(5): 1994-2010.

Kahneman D., Knetsch J.L., and Thaler R.H. 1990. "Experimental Tests of the Endowment Effect and the Coase Theorem," Journal of Political Economy 98: 1325$1348,1326$.

Shah R.C. and Sandvig C. 2008. "Software Defaults as De Facto Regulation: The Case of the Wireless Internet," Information, Communication \& Society 11(1): 25-46, 41.

Thaler R.H. and Sunstein C.R. 2008. Nudge: Improving Decisions about Health, Wealth, and Happiness. New Haven: Yale University Press.

Vaidhyanathan S. 2018. Antisocial Media: How Facebook Disconnects Us and Undermines Democracy. Oxford: Oxford University Press. 


\title{
AOIR
}

Selected Papers of \#AoIR2020: The 21 st Annual Conference of the Association of Internet Researchers Virtual Event / 27-31 October 2020

\section{DISCONNECTING FROM EVERYDAY TECHNOLOGY USE: LESSONS FROM LONG-TERM OUTDOOR ACTIVITIES}

\author{
Airi Lampinen \\ Stockholm University \\ Pedro Ferreira \\ IT-University of Copenhagen

\section{Introduction}

One way in which we can disconnect from networked technologies is by radically altering our engagement with, and being in, the world. In our ongoing research, we have turned to long-term outdoor activities, such as bike touring and hiking, as an empirical site that can inspire fresh ways of conceptualizing how people relate with technology. What happens to routine and habitual uses of technology in a context that is crafted to allow for the enjoyment of disconnection and away-ness from everyday life?

\section{Disconnection and demands communicated through technology}

The outdoors as an idea has long served efforts to rethink how people relate with technology, often drawing on the idea of nature as an escape from modern life. Discussions about technology use and non-use (e.g. Baumer et al., 2015) point to some of the contradictions that individuals face in relating to digital technologies. Harmon and Mazmanian (2013) argue that discourses about disconnection pose "unreasonable expectations of agency and action on both the part of technologies and people", calling for more complicated stories of technologies and their relationships with values in conversations, publications, and future designs.

Connecting closely with our empirical case, Harmon (2015) questions whether the overuse of technology is really a problem of technology, or rather a problem with balancing work and life. She points out that we lack a serious questioning of the 'political economy' of technology and work. In brief, the problem might not be overuse of technology per se, but rather how work (and the broader economic situation) put demands on us - and how those demands come to be communicated through technology. As with Simmel's famous discussion of the clock in metropolitan life, it was 
not that the physical clock itself restructured the modern world but rather that clock time came to be used to routinize and intensify the ordinary lives of city inhabitants.

\section{Case Study: Technology Use in Long-Term Outdoor Activities}

Instead of addressing non-use with the starting point of self-regulation, or nudging people to constrain their digital engagement, our empirical research turns to experiences of 'being outdoors' which are commonly experienced as being away from everyday life, including its routines, pressures, and habitual uses of technology. Our goal with this research is to examine how technology use gets recontextualized and what that might imply for the theorizing of disconnection more broadly. We focused on long-term activities as we suspected these would entail a more radical reworking of both technology use and, more broadly, everyday life.

We conducted in-depth, semi-structured, individual interviews with 24 participants between April 2018 and March 2019. The interviews took place over video calls whenever possible, with voice calls as a backup option. They ranged from just over 30 minutes to over three hours, with most in the range of 50-80 minutes. All interviews were audio-recorded and transcribed verbatim. They are being collaboratively analyzed by the authors through an iterative, material-driven process. In the following, we discuss initial findings regarding two broad themes: (1) experiences of being away and (2) practices of managing disconnection while away. Our examples here focus on bike touring.

\section{Findings: Managing Disconnection While Away}

Firstly, the ways in which participants orient their lives and bodies towards long-term outdoor activities implies significant restructuring and reorganization of their digital ecosystems. Participants often described the value of bike touring precisely in how it disrupted everyday routines by establishing others, summed by one participant, Jeff, in this way: "I mean, the routine is pretty simple. You just wake up, camp, and ride all day. Eat food when you need it. Set up camp and just enjoy each other's company." The forms of disconnection that participants cherished were mainly not attained through purposeful self-discipline, but rather by re-orienting routines towards activities that resist the constant use of digital technologies. The attention-seeking designs characteristic of digitally connected technologies, are out of place in the context of all-consuming yet enjoyable activities like bike touring. Hence, their use simply becomes less relevant for the duration of such activities.

Secondly, and in line with Hesselberth's (2018) reflections on the paradoxes of disconnection, disconnection only exists in relation to being connected. We found that participants managed disconnection to create an enjoyable space of away-ness while connectivity still enabled their journeys by allowing them to accommodate important commitments while away. Being outdoors was, for the most part, actively, and explicitly, designed as time away from the digital demands of social media or messaging 
applications. While participants expressed a broad desire to disconnect, for them, outdoor experiences were not a matter of abstaining completely from technology use. Rather, they sought to balance purposeful disconnection with limited connectivity.

Finally, bike touring, given its duration and physicality, is not an activity that can be done part-time. It forces a reorientation of most aspects of life and requires deliberate efforts to carve out space and time. Technologies are designed often in ways that assume and enforce particular forms of living on their users. Wajcman's study of calendar designers in Silicon Valley (2019) makes a compelling argument of how the designing of time in our technologies structures and determines how we organize ourselves. Bike touring provokes quite a significant split with how technologies tend to organize work and everyday lives. Our participants needed to re-negotiate their digital standing, which was both a burden, but also precisely what they cherished in bike touring. Considering bike touring also presents us with an opportunity to better understand - and even design for - different relations to work and disconnection. We need to challenge our assumptions on what constitutes work and how much of our work rhythms and routines are built in to the technologies we use, including those that we do not even consider to be technologies for 'work'.

\section{Conclusion}

Outdoor activities are commonly presented as an escape from our technology-infused world. In contrast, our interviews reveal experiences that are heavily dependent on technology, both digital and not. However, digital technology is reconfigured when taken out of its ordinary, often urban and indoor, context. Long-term outdoor activities are thus particularly well positioned to reveal how digital technologies can serve to lock us into traditional work rhythms but also enable breaking away from them (Ferreira et al., 2019). By illustrating what happens when everyday rhythms are disrupted, these activities allow us to imagine more purposeful and diverse ways of managing (dis)connection (Helms et al., 2019).

\section{Acknowledgements}

We thank our colleagues Karey Helms and Barry Brown who have worked with us on collecting the empirical materials for this project and co-authored related publications. This work has been supported by the Swedish Foundation for Strategic Research project RIT15-0046.

\section{References}

Baumer, E. P., Burrell, J., Ames, M. G., Brubaker, J. R., \& Dourish, P. 2015. On the importance and implications of studying technology non-use. interactions, 22(2), 52-56.

Ferreira, P., Helms, K., Brown, B., \& Lampinen, A. 2019. From nomadic work to nomadic leisure practice: A study of long-term bike touring. Proceedings of the ACM on Human-Computer Interaction, 3(CSCW), 1-20. 
Harmon, E., \& Mazmanian, M. 2013. Stories of the Smartphone in everyday discourse: conflict, tension \& instability. In Proceedings of the SIGCHI conference on human factors in computing systems (pp. 1051-1060).

Harmon, M. E. 2015. Computing as context: Experiences of dis/connection beyond the moment of non/use. Ph.D. Dissertation. UC Irvine.

Helms, K., Ferreira, P., Brown, B., \& Lampinen, A. 2019. Away and (Dis) connection: Reconsidering the use of digital technologies in light of long-term outdoor activities. Proceedings of the ACM on Human-Computer Interaction, 3(GROUP), 1-20.

Hesselberth P. 2018. "Discourses on Disconnectivity and the Right to Disconnect," New Media \& Society 20(5): 1994-2010.

Wajcman, J. 2019. How Silicon Valley sets time. New Media \& Society, 21(6), 12721289 . 


\title{
AOIR
}

Selected Papers of \#AoIR2020:

The $21_{\text {st }}$ Annual Conference of the

Association of Internet Researchers

Virtual Event / 27-31 October 2020

\section{REPURPOSING UX DESIGN DECKS: INTERFACE CRITICISM, DARK PATTERNS AND THE DESIRE TO DISCONNECT}

\author{
Michael Dieter \\ University of Warwick
}

This paper discusses an interdisciplinary research project that explores the use of card decks in user experience design (UX) practice, and the possibilities of repurposing them as a means to reflect on the problems of social media disconnection. Card decks are variously used as a way to generate insights and assist with problem-solving (Wölfel and Merritt, 2013). They are thought to aid in lateral thinking and are increasingly used to aid innovation. From method cards from agencies like IDEO (2003), seminal 'creative constraints' decks like Brian Eno and Peter Schmidt's Oblique Strategies: Over One Hundred Worthwhile Dilemmas (1975) or decks to standardize government digital services like 18F in the United States (2015), these methodological systems have gained increasing influence with the valorisation of creativity and design as a means to address a wide array of issues. With this general trend, moreover, they have become a conduit for embedding behavioural and psychological 'expertise' into the design of software products and services. In this way, they have become important resources for commercial cultures of connectivity and the 'psycho-computational complex' (Stark 2018), even while also initiating new potential modes of participation, organisation and ways of working collectively.

The use of cards by artists, intellectuals and writers to produce creative work, nevertheless, has a deeper history. As media historians like Peter Krapp (2018) and Markus Krajewski (2011) have shown, these systems have long been a mainstay of intellectual equipment. While state bureaucracies and libraries have used card indexes for purposes of stabilizing knowledge, philosophers and theorists from Hegel to Barthes developed card systems to unsettle their established ways of thinking, serving at once as a memory device and channel for creativity based on idiosyncratic collection criteria, alongside epistemologies of play and chance. With the emphasis on innovative thinking in the Enlightenment era, such document systems were typically positioned as being 'off-the-record' or background materials (indeed, Schmidt's precursor deck to Oblique Strategies was even called 'The Thoughts Behind the Thoughts'). In this respect, as Krapp notes, cards were often considered as a 'private infrastructure' that remained hidden and whose presence easily troubled the image of the spontaneously original, 
autonomous author. Within networked conditions of informational production, however, these presumptions and relations have notably shifted, as these systems frequently take on a more explicitly 'open' and collective role. In doing so, they have been made productive as a creativity dispositif (Reckwitz 2017), whilst shaping the design of software that further challenges these prior notions of invention and discovery.

Contemporary UX card decks, accordingly, maintain an ambiguous potential for unsettling established ways of doing and knowing that cuts across mainstream design practice. The use of cards for design can, for instance, be found in the Scandinavian tradition of participatory design, where contributions from prospective users of a system are solicited through 'pre-digital' paper means with a commitment to democracy and agonistic exchange (Gregory 2003). Yet such methods are also readily apparent in design thinking paradigms, where they primarily serve to identify untapped market opportunities and reinforce new hierarchies of labour (Irani 2018). The use of design pattern decks can, in a similar way, effectively assist with the dissemination of technical expertise and 'know-how', yet also are frequently a resource for 'growth hacking' and maximising user engagement in ways that encourage compulsive use and distraction (Schüll 2014; Lovink 2018). Such decks, in other words, bring together a wide range of socio-technical issues attached to UX practice and, accordingly, additionally provide an ideal means for exploring difficult ethico-political questions for cultures of connection, while speculating on alternatives.

This paper draws from two recent workshops held at the Centre for Interdisciplinary Methodologies (CIM) at University of Warwick and the 2020 Transmediale Festival in Berlin that aimed precisely to reflect on such issues in this domain of creative practice, particularly to consider the epistemologies, technologies and techniques that inform the design of captivating interface experiences. Inspired by recent proposals to develop new styles of interface criticism (Andersen \& Pold 2018; Hadler \& Haupt 2016), the workshops considered what would it mean to retrieve the collectivising potential of such decks for purposes of negation, rather than generating the Next Big Thing. That is, what would it mean to repurpose 'creative' decks for critical reflection or even collective disconnection strategies away from logics aimed at driving engagement, capturing attention and prefiguring experience? The paper will present one output from these sessions in the form of a 'shedding-type' card game titled Disconnect! that foregrounds deceptive UX lock-in strategies known as 'dark patterns' and counterposes these with other well-known tropes of platform engagement (i.e. likes, follows, replies) (Karppi 2018. If disconnection is understood as Mouffean 'constitutive outside' (Hesselberth 2018), then the deck is a means to discuss connective design techniques, while reflecting on desires to escape. It ultimately is offered as an invitation for further experiments in post-digital design, especially to support more empowering paths away from the current configurations of platform capitalism and its onto-epistemologies of control. 


\section{References}

Andersen, C.U., and S. Pold. 2018. The Metainterface: The Art of Platforms, Cities, and Clouds. Cambridge, MA: MIT Press.

Hadler, F. and J. Haupt (eds). 2016. Interface Critique. Berlin: Kulturverlag Kadmos.

Hesselberth P. 2018. "Discourses on Disconnectivity and the Right to Disconnect," New Media \& Society 20(5): 1994-2010.

Irani, L. 2018. “Design Thinking”: Defending Silicon Valley at the Apex of Global Labor Hierarchies', Catalyst Journal.

https://catalystjournal.org/index.php/catalyst/article/download/29638/pdf/.

Gregory, J. 2003. 'Scandinavian Approaches to Participatory Design', International Journal of Engineering Education 19(1): 62-74.

Karppi, T. 2018. Disconnect: Facebook's Affective Bonds. Minneapolis: University of Minnesota Press.

Krajewski, M. 2011. Paper Machines: About Cards \& Catalogs, 1548-1929. Trans. Peter Krapp. Cambridge, MA: MIT Press.

Krapp, P. 2018. 'Paper Slips: The Long Reign of the Index Card and Card Catalog.' In The Routledge Companion to Media Technology and Obsolescence, New York.

Lovink, G. 2019. Sad By Design: On Platform Nihilism. Cambridge: Pluto Press.

Schüll, N. D. 2014. Addiction by Design: Machine Gambling in Las Vegas. Princeton, $\mathrm{NJ}$ : Princeton University Press.

Stark, L. 2018. 'Algorithmic Psychometrics and the Scalable Subject', Social Studies of Science 48(2): 204-31.

Wölfel, C., and T. Merritt. 2013. 'Method Card Design Dimensions: A Survey of CardBased Design Tools.' in Human-Computer Interaction - INTERACT 2013, edited by P. Kotzé, G. Marsden, G. Lindgaard, J. Wesson, and M. Winckler, Springer Berlin Heidelberg, pp. 479-486. 


\title{
AOIR
}

Selected Papers of \#AoIR2020:

The $21_{\text {st }}$ Annual Conference of the

Association of Internet Researchers

Virtual Event / 27-31 October 2020

\section{ETHICS AND EXPERIMENTATION IN THE LIGHT PHONE AND GOOGLE WELLBEING}

\author{
Alex Beattie \\ Victoria University of Wellington \\ Aleena Chia \\ Simon Fraser University
}

The Light Phone is a monochromatic mobile device with an e-ink display and minimal smart functions that is "designed to be used as little as possible." As a consumer electronic in its second iteration, The Light Phone focuses on core utilities such as calls, texts, and maps; the device eschews feeds, social media, advertisements, news and email. According to its designers, The Light Phone respects users' time and attention, cultivating intentional use over infinite scrolls, happiness over connection. Within this promotional imaginary, "Going Light" is a branded lifestyle populated by racially diverse users, art and crafts, slow food and communal meals, family and the outdoors-where the texture and tactility of The Light Phone blend seamlessly into a nostalgic lifeworld of roller-skates, ceramic mugs, Polaroids, and vinyl records. Using the problematic of disconnective experimentation, this paper compares The Light Phone's brand of lightness with the ethics of balance in Google's Digital Wellbeing experimental apps. Both these disconnective experiments are emergent from a culture of connectivity: both evoke the tactility of paper, the aesthetics of minimalism, and the affects of nostalgia. However, while Google's experiments make disconnection esoteric, The Light Phone experiment makes disconnection mundane. By analyzing how the ethics of disconnective experimentation are institutionally, aesthetically, and politically intertwined, this paper presents a framework for the relational assessment of different experimental interventions.

This framework is informed by textual analyses of The Light Phone's promotional materials and two experimental apps from Google's Digital Wellbeing Collection by design and invention consultancy studio Special Projects. According to Google, this collection "showcase ideas and tools that help people find a better balance with technology." The first app "Paper Phone" uses an android app to create a paper version of one's phone on a folded sheet of paper, which includes favorite contacts, maps and meetings, and even activities like recipes and phrasebooks. The second experiment 
"Envelope" is a paper sleeve that transforms the Google Pixel phone into a singlefunction device either for making phone calls or taking photos. This envelope must be torn open to restore the phone's original multi-functions. According to Special Projects, Envelope was inspired by basic phones with limited functions as a periodic alternative to a primary smartphone, which was the original rationale for The Light Phone. In fact, The Light Phone was developed through a partnership between an artist and product designer who met in a Google experimental program in 2014. With these institutional links in mind, textual analyses have been contextualized by a developer interview with The Light Phone's artist.

Google Digital Wellbeing Experiments may have a similar look and feel as The Light Phone. They may even share similar institutional incubation pathways. But their temporal deployments and politics of disconnection are different. As artistic interventions, the resistive potential of both these projects lies not in overt political manifestations, but in their reconfiguration of the doable and visible by creating experiential openings for instability and ambiguity (Beyes and Steyaert 2011). Google's experiments such as Paper Phone and Envelope temporally limit disconnection to a momentary gimmick, thereby inoculating criticisms about the harms of constant connectivity and reinforcing the norm of connectivity. These Experiments operate under Google's Digital Wellbeing - tools that facilitate self-tracking and restricting screen time across a number of its devices, applications, and online platforms. This inoculation is a form of media prophylaxis, which emphasizes harm reduction through self-care discourses that shift responsibility onto the individual without addressing the underlying cultural logics and business models of attention, engagement, and advertising (Mulvin 2018).

By providing a platform for disconnective experiments and tools, Google Digital Wellbeing's message of balance through calibrated connectivity can be understood as part of Silicon Valley's institutionalization of tech ethics. Within this social understanding of ethics, experiments attempt to resolve critical external normative claims about the core logics of the tech industry but do so while fully embedded within those logics (Metcalf, Moss, and boyd 2019). By framing problems of constant connectivity within the language of tools for individual balance and calibration, Google Digital Wellbeing centers ethics in the practices of technologists, not in social worlds or social contracts they develop technical systems for and within. These experiments rationalize the resistive potential of artistic endeavors into frameworks of organizational risk assessment. Furthermore, by incorporating and inoculating against critique about the harms of constant connection, these experiments are a way in which capitalism reshapes itself into less-objectionable forms, thus increasing resistance to similar critiques in the future (Boltanski and Chiapello 2005).

Even though Google's Envelope is inspired by The Light Phone, and both experiments operate at the margins of the culture of connection, the scale of their interventions differ significantly. Envelope creates an experiential gap in the fabric of connection. The Light Phone turns that fabric inside out to reveal alternative configurations of time, 
technology, and connectivity, which are mediated by class and taste. Instead of the corporate logics of ethics, The Light Phone operates through conspicuous nonconsumption: a form of lifestyle politics drawing from consumer activism and neoliberal entrepreneurial selfhood that emphasizes creativity, empowerment, and flexibility (Portwood-Stacer 2013). The Light Phone is a world-building device that envisions a lifestyle and performs an ethos. It is an artistic intervention that creates experiential apertures for destabilizing the norms of connection. While Envelope packages disconnection as a concept and an experiential lapse, The Light Phone evokes disconnection as a sensation that is somewhere between vision and memory. Yet, as a consumer electronic with an affordable mobile coverage plan, it also has the tangibility and practicality for instantiating some glimpse of that vision in everyday life. This experimental potential is, however, entangled in the power-chronography of global circuits of digital labor (Sharma 2014) and class politics of conspicuous nonconsumption.

Crucially, design experiments such as Google's Envelope and The Light Phone may seem incommensurable with the culture of connectivity, yet these experiments and other contemporary disconnection practices emerge from and are constitutive of that culture. These experiments with disconnection are part of the "constitutive outside" of the culture of connectivity (Hesselberth 2018). Understanding and assessing the equivocality of this constitutive outside will require weighing different disconnection practices against each other to understand their connections to each other, so that we may advocate some over others.

\section{References}

Beyes, T. and Steyaert, C. 2011. "The Ontological Politics of Artistic Interventions: Implications for Performing Action Research." Action Research 9, no. 1 (March 2011): $100-115$.

Boltanski, L., and Chiapello, E. 2005. The New Spirit of Capitalism. London ; New York: Verso.

Hesselberth P. 2018. "Discourses on Disconnectivity and the Right to Disconnect," New Media \& Society. 20(5): 1994-2010.

Metcalf, J., Emanuel Moss, and danah boyd. 2019. Owning Ethics: Corporate Logics." Silicon Valley, and the Institutionalization of Ethics. Social Research 86(2): 449-476.

Mulvin, D. 2018. "Media prophylaxis: Night modes and the politics of preventing harm." Information \& Culture. 53(2): 175-202.

Portwood-Stacer, L. 2013. "Media refusal and conspicuous non-consumption: The performative and political dimensions of Facebook abstention." New Media \& Society 15(7): 1041-1057. 
Sharma, S., 2014. In the meantime: Temporality and cultural politics. Duke University Press. 